\title{
The effect of behaviour change techniques on interventions promoting components of a Mediterranean diet among adults of retirement age
}

\author{
J. Lara ${ }^{1,3}$, E. Evans ${ }^{2,3}$, N. Hobbs ${ }^{4}$, T. D. Meyer ${ }^{5}$, F. F. Sniehotta ${ }^{4}$ and J. C. Mathers ${ }^{1,3}$ \\ ${ }^{1}$ Human Nutrition Research Centre, ${ }^{2}$ Clinical Ageing Research Unit, ${ }^{3}$ Institute for Ageing and Health, ${ }^{4}$ Institute of Health \\ and Society, ${ }^{5}$ Institute of Neuroscience, Newcastle University. Biomedical Research Building, Campus for Ageing and \\ Vitality. Newcastle Upon Tyne, NE4 5PL, UK
}

This study is part of LiveWell, a research programme to develop lifestyle-based interventions to improve healthy ageing at the periretirement period. We undertook a systematic review of randomised controlled trials (RCTs) that prospectively evaluated the adoption of a Mediterranean diet or any of its food-group components on healthy or "at risk" middle aged adult subjects. The protocol for the review has been registered with PROSPERO International prospective register of systematic reviews (CRD42011001484). A search of ten databases until December 2010 produced 7480 papers. After abstract and full paper screening, forty-two papers reporting on 18 studies met our criteria of inclusion i.e. RCT studies reporting a mean or median age between 55 and 70 years, reporting food consumption after at least 3 months follow up, and with a country of origin classified as high human development by the United Nations Development Programme. The present analysis explores the association of the CALO-RE taxonomy of standardised behaviour change techniques $(\mathrm{BCTs})^{(1)}$ with fruit and vegetable $(\mathrm{F} \& \mathrm{~V})$ consumption in the reviewed studies.

Twenty-two out of the 40 BCTs listed in the CALO-RE taxonomy were identified and coded in the papers reviewed. The number of BCTs coded per study ranged from 0 to 16 , with a mean of 7 BCTs $(S D=3)$. The most commonly found BCTs were "Provide information on consequences of behaviour in general" and "Goal setting (behaviour)", followed by "Provide instruction on how to perform the behaviour", "Provide information on consequences of behaviour to the individual" and "Goal setting (outcome)".

Meta-regression analysis showed that the number of BCTs per study was not significantly associated $(p=0.174)$ with the effect of interventions. Moderator analysis according to the presence of behaviour change theory as well as by the BCTs identified, showed that studies using the "Barrier identification/ problem solving", "Self-monitoring", "Plan social support/social change", "Use of follow-up prompts", "Goal setting (outcome)", and "Model demonstration" BCTs produced greater effects of interventions on F\&V consumption compared with studies not using these BCTs. Similarly, the presence of behaviour change theories was significantly associated with intervention effectiveness. Overall, this study has identified potentially useful BCTs for implementation in dietary interventions directed to older adults.

The LiveWell project is funded by the Lifelong Health and Wellbeing Cross-Council Programme initiative in partnership with the UK Health Department: The LLHW Funding Partners are: Biotechnology and Biological Sciences Research Council, Engineering and Physical Sciences Research Council, Economic and Social Research Council, Medical Research Council, Chief Scientist Office of the Scottish Government Health Directorates, National Institute for Health Research /The Department of Health, The Health and Social Care Research \& Development of the Public Health Agency (Northern Ireland), and Wales Office of Research and Development for Health and Social Care, Welsh Assembly Government.

1. Michie S, Ashford S, Sniehotta FF, Dombrowski SU, Bishop A, French DP (2011) A refined taxonomy of behaviour change techniques to help people change their physical activity and healthy eating behaviours: the CALO-RE taxonomy. Psychol Health 26, 1479-1498. 